Pacific Journal of Mathematics

SOME PROPERTIES OF A SPECIAL SET OF RECURRING C. who ss 


\title{
SOME PROPERTIES OF A SPECIAL SET OF RECURRING SEQUENCES
}

\author{
H. C. WilliamS
}

\begin{abstract}
Several number theoretic and identity properties of three special second order recurring sequences are established. These are used to develop a necessary and sufficient condition for any integer of the form $2^{n} 3^{m} A-1\left(A<2^{n+1} 3^{m}-1\right)$ to be prime. This condition can be easily implemented on a computer.
\end{abstract}

1. Introduction. Various tests for primality of integers of the form $2^{n} A-1$ and $3^{n} A-1$ are currently available; for example, Lehmer [2] and Riesel [5] have developed necessary and sufficient conditions for $2^{n} A-1$ to be prime when $A<2^{n}$ and Williams [6] has given a necessary and sufficient condition for the primality of $2 A 3^{n}-1$ when $A<4 \cdot 3^{n}-1$. Of special concern to Riesel was the determination of the primality of $3 A 2^{n}-1$; in this paper we present a simple necessary and sufficient condition for $2^{n} 3^{m} A-1$ to be prime when $A<2^{n+1} 3^{m}-1$. In order to obtain this result we must first develop some properties of a special set of second order linear recurring sequences.

Let $a, b$ be two integers and put $\alpha=a+b \rho, \beta=a+b \rho^{2}$, where $\rho^{2}+\rho+1=0$. We define for any integer $n$

$$
\begin{aligned}
& R_{n}=\frac{\rho \alpha^{n}-\rho^{2} \beta^{n}}{\rho-\rho^{2}}, \\
& S_{n}=\frac{\rho^{2} \alpha^{n}-\rho \beta^{n}}{\rho-\rho^{2}}, \\
& T_{n}=\frac{\alpha^{n}-\beta^{n}}{\rho-\rho^{2}} .
\end{aligned}
$$

We see that $R_{0}=1, S_{0}=-1, T_{0}=0, R_{1}=a-b, S_{1}=-a, T_{1}=b$. Putting $G=\alpha+\beta=2 a-b$ and $H=\alpha \beta=a^{2}-a b+b^{2}$, we get

$$
\begin{aligned}
& R_{n+2}=G R_{n+1}-H R_{n}, \\
& S_{n+2}=G S_{n+1}-H S_{n}, \\
& T_{n+2}=G T_{n+1}-H T_{n} .
\end{aligned}
$$

It follows that $R_{n}, S_{n}, T_{n}$ are integers for any nonnegative integral value of $n$.

In the next sections of this paper we present a number of identities satisfied by the $R_{n}, S_{n}, T_{n}$ functions. We also develop some of their number theoretic properties. It should be noted that 
the function $T_{n}$ is simply a constant multiple $b$ of the Lucas function $U=\left(\alpha^{n}-\beta^{n}\right) /(\alpha-\beta)$; hence, many of its properties are easily deduced from the well-known (see, for example, [2]) properties of the Lucas functions.

2. Some identities. We first note that from the definition of $R_{n}, S_{n}, T_{n}$, we obtain the fundamental identity

$$
R_{n}+S_{n}+T_{n}=0 \text {. }
$$

We can easily verify for any integers $m, n$ that

$$
\begin{aligned}
& R_{m+n}=R_{m} R_{n}-T_{m} T_{n}, \\
& S_{m+n}=T_{m} T_{n}-S_{m} S_{n}, \\
& T_{m+n}=S_{m} S_{n}-R_{m} R_{n}=T_{m} R_{n}-S_{m} T_{n}=R_{m} T_{n}-T_{m} S_{n} .
\end{aligned}
$$

Putting $m=1$, we get

$R_{n+1}=a R_{n}+b S_{n}, \quad S_{n+1}=(a-b) S_{n}-b R_{n}, \quad T_{n+1}=(b-a) R_{n}-a S_{n}$.

Putting $n=m$, we see that

$$
\begin{gathered}
R_{2 n}=-S_{n}\left(2 R_{n}+S_{n}\right), \quad S_{2 n}=R_{n}\left(2 S_{n}+R_{n}\right), \\
T_{2 n}=T_{n}\left(R_{n}-S_{n}\right) ;
\end{gathered}
$$

also, by using these results and putting $m=2 n$ above, we get

$$
\begin{gathered}
R_{3 n}=S_{n}^{3}-3 S_{n} R_{n}^{2}-R_{n}^{3}, \quad S_{3 n}=R_{n}^{3}-3 S_{n}^{2} R_{n}-S_{n}^{3}, \\
T_{3 n}=-3 R_{n} S_{n} T_{n}=-\left(R_{n}^{3}+S_{n}^{3}+T_{n}^{3}\right) . \quad\left(\text { Use }-R_{n}^{3}=\left(S_{n}+T_{n}\right)^{3} .\right)
\end{gathered}
$$

Since

$$
H^{n} R_{-n}=-S_{n}, \quad H^{n} S_{-n}=-R_{n}, \quad H^{n} T_{-n}=-T_{n},
$$

it follows that

$$
\begin{gathered}
H^{m} R_{n-m}=T_{m} T_{n}-S_{m} R_{n}, \quad H^{m} S_{n-m}=R_{m} S_{n}-T_{m} T_{n}, \\
H^{m} T_{n-m}=S_{m} R_{n}-R_{m} S_{n}=R_{m} T_{n}-T_{m} R_{n}=T_{m} S_{n}-R_{m} T_{n} .
\end{gathered}
$$

If, in the first of these formulas, we put $n=m$, we have $R_{0} H^{n}=$ $T_{n}^{2}-R_{n} S_{n}$; hence, we can deduce the following:

$$
\begin{gathered}
T_{n}^{2}-R_{n} S_{n}=R_{n}^{2}-T_{n} S_{n}=S_{n}^{2}-T_{n} R_{n}=H^{n} \\
T_{n}^{2}+R_{n} T_{n}+R_{n}^{2}=R_{n}^{2}+S_{n} R_{n}+S_{n}^{2}=S_{n}^{2}+T_{n} S_{n}+T_{n}^{2}=H^{n} \\
T_{n} S_{n}+S_{n} R_{n}+R_{n} T_{n}=-H^{n}
\end{gathered}
$$

More generally, we have 


$$
\begin{aligned}
& R_{n}^{2}-R_{n-m} R_{n+m}=S_{n}^{2}-S_{n-m} S_{n+m}=T_{n}^{2}-T_{n-m} T_{n+m}=H^{n-m} T_{m}^{2}, \\
& R_{n}^{2}-T_{n-m} S_{m+n}=S_{n}^{2}-R_{n-m} T_{m+n}=T_{n}^{2}-S_{n-m} R_{n+m}=H^{n-m} R_{m}^{2}, \\
& R_{n}^{2}-S_{n-m} T_{n+m}=S_{n}^{2}-T_{n-m} R_{m+n}=T_{n}^{2}-R_{n-m} S_{m+n}=H^{n-m} S_{m}^{2} .
\end{aligned}
$$

We also have

$$
\begin{gathered}
R_{n+m}^{2}-H^{2 m} R_{n-m}^{2}=T_{2 m} S_{2 n}, \quad S_{n+m}^{2}-H^{2 m} S_{n-m}^{2}=T_{2 m} R_{2 n}, \\
T_{n+m}^{2}-H^{2 m} T_{n-m}^{2}=T_{2 m} T_{2 n} .
\end{gathered}
$$

A great many other identities satisfied by these functions can be developed; for example, since

$$
R_{n}+S_{n}+T_{n}=0, \quad R_{n} S_{n}+S_{n} T_{n}+R_{n} T_{n}=-H^{n},
$$

we can use Waring's formula (see, for example, [4] p. 5) to obtain

$$
\begin{gathered}
R_{n}^{m}+S_{n}^{m}+T_{n}^{m}=\left\{\begin{array}{lr}
\sum_{j=0}^{[r / 3]} \frac{(r-j-1) ! 2 r}{(2 j) !(r-3 j) !} H^{(r-3 j) n}\left(R_{n} S_{n} T_{n}\right)^{2 j} \quad(m=2 r) \\
\sum_{j=0}^{[(r-1) / 3]} \frac{(r-1-j) !(2 r+1)}{(2 j+1) !(r-1-3 j) !} H^{(r-1-3 j) n}\left(R_{n} S_{n} T_{n}\right)^{2 j+1}
\end{array} \quad(m=2 r+1)\right. \\
\left(R_{n} S_{n}\right)^{m}+\left(S_{n} T_{n}\right)^{m}+\left(T_{n} R_{n}\right)^{m} \\
=(-1)^{m} \sum_{j=0}^{[m / 3]}(-1)^{j} \frac{(m-2 j-1) ! m}{(m-3 j) ! j !} H^{n(m-3 j)}\left(R_{n} S_{n} T_{n}\right)^{2 j}
\end{gathered}
$$

for $m>0$. From these we deduce the rather interesting identities

$$
\begin{aligned}
& R_{n}^{4}+S_{n}^{4}+T_{n}^{4}=2 H^{2 n}, \\
& R_{n}^{7}+S_{n}^{7}+T_{n}^{7}=7 H^{2 n} R_{n} S_{n} T_{n}, \\
& R_{n}^{10}+S_{n}^{10}+T_{n}^{10}=2 H^{5 n}+15 H^{2 n} R_{n}^{2} S_{n}^{2} T_{n}^{2}, \\
& R_{n}^{5} S_{n}^{5}+R_{n}^{5} T_{n}^{5}+S_{n}^{5} T_{n}^{5}=5 H^{2 n} R_{n}^{2} S_{n}^{2} T_{n}^{2}-H^{5 n} .
\end{aligned}
$$

The following identities are also of some interest:

$$
\begin{aligned}
& \left(S_{n}\left(S_{n}^{2}-3 H^{n}\right)\right)^{3}+\left(T_{n}\left(T_{n}^{2}-3 H^{n}\right)\right)^{3}+\left(R_{n}\left(R_{n}^{2}-3 H^{n}\right)\right)^{3} \\
& =3\left(R_{n} S_{n} T_{n}\right)^{3} \text {, } \\
& \left(R_{n} S_{n}\left(H^{n}+T_{n}^{2}\right)\right)_{1}^{4}+\left(R_{n} T_{n}\left(H^{n}+S_{n}^{2}\right)\right)^{4}+\left(S_{n} T_{n}\left(H^{n}+R_{n}^{2}\right)\right)^{4} \\
& =H^{8 n}+28 H^{2 n}\left(R_{n} S_{n} T_{n}\right)^{4} \text {. }
\end{aligned}
$$

Both of these formulas can be derived by expanding the powers of the binomials and using the formulas above for expressions of the form $R_{n}^{j}+S_{n}^{j}+T_{n}^{j}$ and $\left(R_{n} S_{n}\right)^{j}+\left(S_{n} T_{n}\right)^{j}+\left(T_{n} R_{n}\right)^{j}$.

If we put $W_{n}=R_{n}-S_{n}, X_{n}=S_{n}-T_{n}=2 S_{n}+R_{n}, Y_{n}=T_{n}$ $R_{n}=-2 R_{n}-S_{n}$, we have 


$$
\begin{gathered}
W_{n}+X_{n}+Y_{n}=0, \\
3 R_{n}=W_{n}-Y_{n}, \quad 3 S_{n}=X_{n}-W_{n}, \quad 3 T_{n}=Y_{n}-X_{n} \\
R_{2 n}=S_{n} Y_{n}, \quad S_{2 n}=R_{n} X_{n}, \quad T_{2 n}=T_{n} W_{n} .
\end{gathered}
$$

We also have

$$
\begin{aligned}
& 3 W_{m+n}=W_{m} W_{n}+Y_{m} X_{n}+Y_{n} X_{m}, \\
& 3 X_{m+n}=Y_{m} Y_{n}+X_{m} W_{n}+W_{m} X_{n}, \\
& 3 Y_{m+n}=X_{m} X_{n}+Y_{m} W_{n}+W_{m} Y_{n},
\end{aligned}
$$

and from these we are able to derive

$$
\begin{aligned}
& W_{2 n}=\left(W_{n}^{2}+2 X_{n} Y_{n}\right) / 3=X_{n} Y_{n}+H^{n}=W_{n}^{2}-2 H^{n}, \\
& Y_{2 n}=\left(X_{n}^{2}+2 W_{n} Y_{n}\right) / 3=W_{n} Y_{n}+H^{n}=X_{n}^{2}-2 H^{n}, \\
& X_{2 n}=\left(Y_{n}^{2}+2 X_{n} W_{n}\right) / 3=W_{n} X_{n}+H^{n}=Y_{n}^{2}-2 H^{n},
\end{aligned}
$$

and

$$
\begin{aligned}
& 3 X_{3 n}=X_{n}^{3}+3 X_{n}^{2} Y_{n}-Y_{n}^{3}, \\
& 3 Y_{3 n}=Y_{n}^{3}+3 Y_{n}^{2} X_{n}-X_{n}^{3}, \\
& W_{3 n}=X_{n} Y_{n} W_{n} .
\end{aligned}
$$

Many other identities similar to those satisfied by the $R_{n}, S_{n}, T_{n}$ functions are satisfied by $W_{n}, X_{n}, Y_{n}$ functions.

3. Some number theoretic results. In the discussion that follows we will assume that $a$ and $b$ satisfy the following two properties:

$$
\begin{gathered}
(a, b)=1, \\
a \neq \equiv-b(\bmod 3) .
\end{gathered}
$$

It follows from (1) and (2) that $(G, H)=1$. We can now develop several divisibility properties of the $R_{n}, S_{n}, T_{n}$ functions. We will also assume in what follows that $n, m$ represent positive integers.

Lemma 1. For any $n,\left(R_{n}, H\right)=\left(S_{n}, H\right)=\left(T_{n}, H\right)=1$.

Proof. If $p$ is any prime divisor of $R_{n}$ and $H$, then by (1.1) $p$ is a divisor of $R_{n-1}$. By continuing this reasoning, we see that $p \mid R_{1}$. If $p \mid R_{1}$ and $p \mid H$, then $R_{0}=1$ and $p \mid G$, which is impossible. In the same way we see that $\left(S_{n}, H\right)=1$. Also, if $p \mid\left(T_{n}, H\right)$, then by 
the above reasoning $p \mid T_{1}=b$. Since $p \mid H$, we have $p \mid a$ and consequently $p \mid G$.

Lemma 2. For any $n,\left(R_{n}, S_{n}\right)=\left(S_{n}, T_{n}\right)=\left(T_{n}, R_{n}\right)=1$.

Proof. If $p$ is any prime divisor of any two of $R_{n}, S_{n}, T_{n}$, then by (2.4) $p$ must divide $H$, which is impossible by the preceding lemma.

Since $T_{n}$ is a simple multiple of the Lucas function $U_{n},\left\{T_{n}\right\}$ is divisibility sequence, i.e., $T_{n} \mid T_{m}$ whenever $n \mid m$. The analogous properties of $R_{n}$ and $S_{n}$ are given in

THEOREM 1. Suppose $n \mid m$. If $m / n \equiv 1(\bmod 3)$, then $R_{n} \mid R_{m}$ and $S_{n} \mid S_{m} ;$ if $m / n \equiv-1(\bmod 3)$, then $R_{n}\left|S_{m}, S_{n}\right| R_{n} ;$ if $m / n \equiv 0$ $(\bmod 3)$, then $R_{n}\left|T_{m}, S_{n}\right| T_{m}$.

Proof. From the identities of $\S 1$ we see that $R_{n}\left|S_{2 n}, S_{n}\right| R_{2 n}$, $R_{n}\left|T_{3 n}, S_{n}\right| T_{3 n}$. Now since $T_{3 n} \mid T_{3 k n}$,

$$
\begin{aligned}
R_{(3 k+t) n} & =R_{3 k n} R_{t n}-T_{3 k n} T_{t n} \\
& \equiv R_{3 k n} R_{t n}\left(\bmod R_{n} S_{n}\right) .
\end{aligned}
$$

If $t=1, R_{n} \mid R_{(3 k+t) n}$; if $t=2, S_{n} \mid R_{(3 k+t) n}$. The remaining results are proved in a similar manner.

Let $T_{\omega(m)}$ be the first term of the sequence

$$
T_{1}, T_{2}, T_{3}, \cdots, T_{n},
$$

in which $m$ occurs as a factor. We will call $\omega=\omega(m)$ the "rank of apparition" of $m$. From the theory of Lucas functions, it follows that if $m \mid T_{n}$, then $\omega(m) \mid n$ and consequently that $\left(T_{m}, T_{n}\right)=T_{(m, n)}$. We also have the result that if $(H, m)=1$, then $\omega(m)$ always exists.

We now define $\omega_{1}=\omega_{1}(m)$ and $\omega_{2}=\omega_{2}(m)$ as analogues of $\omega(m)$. We say for a given $m$ that $R_{\omega_{1}}$ and $S_{\omega_{2}}$ are respectively the first term of the sequences

$$
\left\{R_{k}\right\}_{k=1}^{\infty} \text { and }\left\{S_{k}\right\}_{k=1}^{\infty} \text { which } m \text { divides. }
$$

It is not in general true that $\omega_{1}(m)$ or $\omega_{2}(m)$ exist for any $m$ such that $(m, H)=1$. In the results that follow we give some characterization of those values of $m$ such that $\omega_{1}(m)$ or $\omega_{2}(m)$ do exist. In Theorems 2, 3, 4, and Lemma 3 we give results concerning $R_{n}$ and $\omega_{1}$ only; however, analogous results involving $S_{n}$ and $\omega_{2}$ for each of these are also true and their proofs are similar.

THEOREM 2. If $(m, H)=1$ and $\omega_{1}$ exists, then $\omega_{2}$ exists, $3 \mid \omega$, $\omega_{1}=\omega / 3$ or $2 \omega / 3$, and $\omega_{1}+\omega_{2}=\omega$. 
Proof. Suppose $\omega_{1} \geqq \omega$. We have

$$
\omega_{1}=q \omega+r \quad\left(0 \leqq r<\omega \leqq \omega_{1}\right)
$$

and

$$
0 \equiv R_{\omega_{1}}=R_{q \omega} R_{r}-T_{q \omega} T_{r} \equiv R_{q \omega} R_{r}(\bmod m) .
$$

Since $m \mid T_{q \omega}$ and $\left(T_{q \omega}, R_{q \omega}\right)=1$, we see that $m \mid R_{r}$, which is impossible. Thus, $\omega_{1}<\omega$.

Since $m \mid T_{3 \omega_{1}}$, we must have $\omega \mid 3 \omega_{1}$; since $\omega>\omega_{1}$, we see that $3 \mid \omega$ and $\omega_{1}=\omega / 3$ or $2 \omega / 3$. Now

$$
H^{\omega_{1}} S_{\omega-\omega_{1}}=S_{\omega} R_{\omega_{1}}-T_{\omega} T_{\omega_{1}} \equiv 0(\bmod m) ;
$$

thus, $m \mid S_{\omega-\omega_{1}}$ and $\omega_{2} \leqq \omega-\omega_{1}<\omega$. Since as with $\omega_{1}, m \mid T_{3 \omega_{2}}$, it follows that $\omega \mid 3 \omega_{2}$, so $\omega_{2}=\omega / 3$ or $2 \omega / 3$. Now if $\omega_{1}=\omega_{2}=\omega / 3$ or $2 \omega / 3$, then $R_{\omega_{1}}+S_{\omega_{1}}+T_{\omega_{1}}=0$ implies $m \mid T_{\omega_{1}}$, which is a contradiction since $\omega_{1}<\omega$. Thus, since $\omega_{1} \neq \omega_{2}$, we must have $\omega_{1}+\omega_{2}=\omega$.

THeOREM 3. If $(m, H)=1$ and $m \mid R_{n}$, then $\omega_{1}$ exists and either $\omega_{1} \mid n$ and $n / \omega_{1} \equiv 1(\bmod 3)$ or $w_{2} \mid n, \omega_{2}=\omega_{1} / 2$ and $n / \omega_{2} \equiv-1(\bmod 6)$.

Proof. Let $n=3 \omega_{1} q+r\left(0 \leqq r<3 \omega_{1}\right)$; then

$$
0 \equiv R_{n}=R_{3 \omega_{1} q} R_{r}-T_{3 \omega_{1} q} T_{r} \equiv R_{3 \omega_{1} q} R_{r}(\bmod m)
$$

and $m \mid R_{r}$. We now distinguish two cases.

Case 1. $\omega_{1}=\omega / 3$. Here we have $r<\omega$ and $3 r<3 \omega$. Since $m \mid T_{3 r}$, we see that $3 r=\omega$ or $2 \omega$. If $3 r=2 \omega$, then $r=\omega_{2}$, which, since $\left(R_{r}, S_{r}\right)=1$, is impossible. Thus, $r=\omega / 3=\omega_{1}, \omega_{1} \mid n$ and $n / \omega_{1} \equiv 1(\bmod 3)$.

Case 2. $\omega_{1}=2 \omega / 3$. In this case we see that $r<2 \omega$ and $3 r<6 \omega$. Thus, $3 r$ is one of $\omega, 2 \omega, 4 \omega, 5 \omega$. If $3 r=\omega$ or $4 \omega$, then $r=\omega_{2}$ or $4 \omega_{2}$. Since $\left(R_{r}, S_{r}\right)=1$, this is impossible. Thus $r=\omega_{1}$ or $\omega+\omega_{1}$. If $r=\omega_{1}$, we have $\omega_{1} \mid n$ and $n / \omega_{1} \equiv 1(\bmod 3)$; if $r=$ $\omega+\omega_{1}$, then $n=3 \omega_{1} q+\omega+\omega_{1}=6 \omega_{2} q+3 \omega_{2}+2 \omega_{2}=(6 q+5) \omega_{2}$.

COROLLARY. Under the conditions of Theorem 3, we must have $n \equiv \omega_{1}\left(\bmod 3^{\nu+1}\right)$, where $3^{\nu} \| \omega_{1}, \nu \geqq 0$.

THEOREM 4. If $m$ and $n$ are integers such that $(m, n)=1$, then $\omega_{1}(m n)$ exists if and only if $\omega_{1}(m)$ and $\omega_{1}(n)$ exist and $\omega_{1}(m) \equiv \omega_{1}(n)$ $\left(\bmod 3^{\nu+1}\right)$, where $3^{\nu} \| \omega_{1}(m), \nu \geqq 0$. 
Proof. Suppose $\Omega_{1}=\omega_{1}(m n)$ exists; then clearly $\omega_{1}=\omega_{1}(m)$ and $\omega_{1}^{*}=\omega_{1}(n)$ exist and

$$
\begin{array}{ll}
\Omega_{1} \equiv \omega_{1}\left(\bmod 3^{\nu+1}\right) & \left(3^{\nu} \| \omega_{1}\right), \\
\Omega_{1} \equiv \omega_{1}^{*}\left(\bmod 3^{\nu^{*}+1}\right) & \left(3^{2^{*}} \| \omega_{1}^{*}\right) .
\end{array}
$$

It follows that $\nu=\nu^{*}$ and $\omega_{1} \equiv \omega_{1}^{*}\left(\bmod 3^{\nu+1}\right)$.

If $\omega_{1}$ and $\omega_{1}^{*}$ exist and $\omega_{1} \equiv \omega_{1}^{*}\left(\bmod 3^{\nu+1}\right)\left(3^{\nu} \| \omega_{1}\right)$, put $\Omega=\left[\omega_{1}, \omega_{1}^{*}\right]$. We see that

$$
\frac{\Omega}{\omega_{1}} \equiv \frac{\Omega}{\omega_{1}^{*}} \not \equiv 0(\bmod 3) \text {. }
$$

If $\Omega / \omega_{1} \equiv 1(\bmod 3)$, then $R_{\Omega} \equiv 0(\bmod m n) ;$ if $\Omega / \omega \equiv-1(\bmod 3)$, then $S_{\Omega} \equiv R_{2 \Omega} \equiv 0(\bmod m n)$. In either case we see that $\omega_{1}(m n)$ must exist.

In order to continue our discussion of the existence of $\omega_{1}(m)$ and $\omega_{2}(m)$ it is necessary to consider the question of the existence of $\omega_{1}\left(p^{n}\right), \omega_{2}\left(p^{n}\right)$, where $p$ is a prime. This is done in the next section.

4. Some results modulo $p$. From the theory of Lucas functions we know that if $p^{\lambda}>2$, and $p^{\lambda} \| T_{n}$ then $p^{\lambda+\nu} \| T_{n p^{\nu}}$; also, if $p^{\lambda}=2$ and $2 \mid T_{n}$, then $4 \mid T_{2 n}$. We will attempt to discover similar results for $R_{n}$ and $S_{n}$. We must deal with the special case $p=3$ separately.

LEMMA 3. If $3^{\nu} \| R_{m}$ when $\nu \geqq 1$, then $3^{\nu} \| R_{m n}$ when $n \equiv 1(\bmod 3)$; otherwise, $3 \nmid R_{m n}$.

Proof. Certainly $3^{\nu} \mid R_{m n}$ when $n \equiv 1(\bmod 3)$ (Theorem 1$)$; suppose $3^{\nu+1} \mid R_{m n}$. Now $3^{\nu+2} \mid T_{9 m}$ and $3^{\nu+2} \mid T_{3 m n}$; hence, $3^{\nu+2} \mid T_{3 m}=\left(T_{9 m}, T_{3 m n}\right)$, which is impossible. If $3 \mid R_{m n}$ when $n \neq \equiv 1(\bmod 3)$, then since $3 \mid R_{m}$, we have $3 \mid\left(T_{m}, R_{m}\right)$ or $3 \mid\left(R_{m}, S_{m}\right)$, neither of which is possible.

We deal now with any prime $p \neq 3$.

THEOREM 5. Let $p$ be any prime which is not 3 and suppose $\lambda>1$. If $p^{\lambda} \neq 2$ and $p^{\lambda} \| R_{m}$, then $p^{\lambda+\nu} \| R_{m p^{\nu}}$ when $p^{\nu} \equiv 1(\bmod 3)$ and $p^{\lambda+\nu} \| S_{m p^{\nu}}$ when $p^{\nu} \equiv-1(\bmod 3)$. If $p^{\lambda} \neq 2$ and $p^{\lambda} \| S_{m}$, then $p^{\lambda+\nu} \| S_{m p^{\nu}}$ when $p^{\nu} \equiv-1(\bmod 3)$ and $p^{\lambda+\nu} \| R_{m p^{\nu}}$ when $p^{\nu} \equiv-1(\bmod 3)$. If $2 \mid R_{m}$, then $4 \mid S_{2 m}$; if $2 S_{m}$, then $4 \mid R_{2 m}$.

Proof. From the definitions of $R_{n}$ and $S_{n}$ it is easy to show that

$$
\begin{aligned}
& \rho^{2} S_{m p}-\rho R_{m p}=\left(\rho^{2} S_{m}-\rho R_{m}\right)^{p}, \\
& \rho S_{m p}-\rho^{2} R_{m p}=\left(\rho S_{m}-\rho^{2} R_{m}\right)^{p} .
\end{aligned}
$$


Suppose $p \neq 2$. If $p^{\lambda} \| R_{m}$, then

$$
\begin{aligned}
& \rho^{2} S_{m p}-\rho R_{m p} \equiv \rho^{2 p} S_{m}^{p}-p \rho^{2 p-1} R_{m} S_{m}^{p-1}\left(\bmod p^{\lambda+2}\right), \\
& \rho S_{m p}-\rho^{2} R_{m p} \equiv \rho^{p} S_{m}^{p}-p \rho^{p+1} R_{m} S_{m}^{p-1}\left(\bmod p^{\lambda+2}\right) ;
\end{aligned}
$$

therefore,

$$
R_{m p} \equiv p R_{m} S_{m}^{p-1}\left(\bmod p^{\lambda+2}\right) \quad \text { when } \quad p \equiv 1(\bmod 3)
$$

and

$$
S_{m p} \equiv p R_{m} S_{m}^{p-1}\left(\bmod p^{\lambda+2}\right) \quad \text { when } \quad p \equiv-1(\bmod 3) .
$$

We get similar results when $p^{2} \| S_{m}$. Thus the theorem is true for $\nu=1$. That it is true for a general $\nu$ can be easily shown by induction on $\nu$. When $p=2$ we prove the theorem by using the identities (2.2).

When $p \neq 3$, we see that $\omega_{1}\left(p^{n}\right)$ and $\omega_{2}\left(p^{n}\right)$ both exist when $\omega_{1}(p)$ and $\omega_{2}(p)$ exist. We need now only consider the problem of when $\omega_{1}(p), \omega_{2}(p)$ exist. Since $3 \mid T_{3}$, we see that $\omega_{1}\left(3^{n}\right)$ exists only if $3^{n} \mid R_{1}$ or $3^{n} \mid S_{1}$ and similarly for $\omega_{2}\left(3^{n}\right)$.

Let $p(\neq 3)$ be a prime. If $p \equiv 1(\bmod 3)$, let

$$
\pi=r+s \rho,
$$

where $r \equiv-1(\bmod 3), \quad 3 \mid s$ and $N(\pi)=\pi \bar{\pi}=r^{2}-s r+s^{2}=p ; \quad$ if $p \equiv-1(\bmod 3)$, let $\pi=\bar{\pi}=p, N(\pi)=p^{2}$. We have $\pi$ a prime in the Eisenstein field $Q(\rho)$ and we define $[\mu \mid \pi]$ to the cubic character of $\mu \in Q[\rho]$ modulo $\pi$. That is

$$
\mu^{(N(\pi)-1) / 3} \equiv\left[\frac{\mu}{\pi}\right](\bmod \pi)
$$

and

$$
\left[\frac{\mu}{\pi}\right]=1, \quad \rho, \quad \text { or } \quad \rho^{2}
$$

THEOREM 6. If $p \equiv \varepsilon(\bmod 3)$, where $|\varepsilon|=1$, and $[H \alpha \mid \pi]=\rho^{\eta}$, then $p \mid R_{(p-\varepsilon) / 3}$ when $\eta=2, p \mid S_{(p-\varepsilon) / 3}$ when $\eta=1$, and $\rho \mid T_{(p-\varepsilon) / 3}$ when $\eta=0$.

Proof. We consider two possible cases.

Case 1. $\varepsilon=+1$. In this case $N(\pi)=p$,

$$
\alpha^{p} \equiv \alpha(\bmod p), \quad \text { and } \quad(\alpha H)^{(p-1) / 3} \equiv \rho^{\eta}(\bmod \pi) ;
$$

hence, 


$$
\alpha^{2(p-1) / 3} \beta^{(p-1) / 3} \equiv \rho^{\eta}(\bmod \pi)
$$

and

$$
\alpha^{(p-1) / 3} \equiv \rho^{2 \eta} \beta^{(p-1) / 3}(\bmod \pi) .
$$

The theorem follows easily from this result and the definition of $R_{n}, S_{n}$ and $T_{n}$.

Case 2. $\varepsilon=-1$. In this case $N(\pi)=p^{2}, \alpha^{p} \equiv \beta(\bmod p)$,

$$
(\alpha H)^{\left(p^{2}-1\right) / 3} \equiv \alpha^{\left(p^{2}-1\right) / 3} \equiv\left(\alpha^{p-1}\right)^{(p+1) / 3} \equiv(\beta / \alpha)^{(p+1) / 3}(\bmod p) .
$$

It follows that

$$
\alpha^{(p+1) / 3} \equiv \rho^{2 \eta} \beta^{(p+1) / 3}(\bmod p) .
$$

If $\eta=0$ and $p \not \equiv \varepsilon(\bmod 9)$, then $\omega_{1}(p)$ and $\omega_{2}(p)$ can not exist; for, in this case, $\omega \mid(p-\varepsilon) / 3$ and $3 \nmid \omega$. If, on the other hand, $\eta \neq 0$, then $\omega_{1}$ and $\omega_{2}$ do exist and

$$
\begin{aligned}
& \omega_{1} \equiv 2 \eta(p-\varepsilon) / 3\left(\bmod 3^{\nu}\right) \\
& \omega_{2} \equiv \eta(p-\varepsilon) / 3\left(\bmod 3^{\nu}\right)
\end{aligned}
$$

where $3^{2} \| p-\varepsilon$. The question of whether $\omega_{1}=2 \omega_{2}$ or $\omega_{1}=\omega_{2} / 2$ seems to be rather difficult. We can give some simple results on this but we first require

THEOREM 7. If $p$ is a prime such that $p \equiv \varepsilon(\bmod 6),|\varepsilon|=1$, $\lambda=(p-\varepsilon) / 6$, and $\sigma=(H \mid p)$ (Legendre symbol), then one and only one of $W_{\lambda}, X_{\lambda}, Y_{\lambda}, R_{\lambda}, S_{\lambda}, T_{\lambda}$ is divisible by $p$ and that one is given in the table below according to the value of $\sigma$ and $\eta$.

\begin{tabular}{|c|c|c|c|}
\hline$\sigma$ & 0 & 1 & 2 \\
\hline-1 & $W_{\lambda}$ & $X_{\lambda}$ & $Y_{\lambda}$ \\
\hline 1 & $T_{\lambda}$ & $R_{\lambda}$ & $S_{\lambda}$ \\
\hline
\end{tabular}

Proof. If $\varepsilon=1, \alpha^{p-\varepsilon} \equiv \beta^{p-\varepsilon} \equiv 1(\bmod p)$; if $\varepsilon=-1, \alpha^{p-\varepsilon} \equiv \beta^{p-\varepsilon} \equiv$ $\alpha \beta=H(\bmod p)$; hence, we easily obtain the result that

$$
R_{6 \lambda} \equiv H^{(1-\varepsilon) / 2}, \quad S_{6 \lambda} \equiv-H^{(1-\varepsilon) / 2}, \quad T_{6 \lambda} \equiv 0(\bmod p) .
$$

Thus, $W_{6 \lambda} \equiv 2 H^{(1-\varepsilon) / 2}$ and

$$
2 H^{(1-\varepsilon) / 2} \equiv W_{3 \lambda}^{2}-2 H^{(p-\varepsilon) / 2} \equiv W_{3 \lambda}^{2}-2 \sigma H^{(1-\varepsilon) / 2}(\bmod p) .
$$

If $\sigma=-1$, then $p \mid W_{3 \lambda}$ and since 


$$
W_{n}^{2}+3 T_{n}^{2}=4 H^{n},
$$

$p \nmid T_{3 \lambda}$. Now $p \mid W_{\lambda} X_{\lambda} Y_{\lambda}$ and the prime $p$ can divide only one of $W_{\lambda}$, $X_{\lambda}$ or $Y_{\lambda}$; for, if it divided any two of these it would divide the third. It follows that it would also divide $R_{\lambda}, S_{\lambda}$, and $T_{\lambda}$, which is impossible. If $p \mid W_{\lambda}$, then $p \mid T_{2 \lambda}$ and $\eta=0$; if $p \mid X_{\lambda}$, then $p \mid S_{2 \lambda}$ and $\eta=1$; if $p \mid Y_{\lambda}$, then $p \mid R_{2 \lambda}$ and $\eta=2$.

If $\sigma=1$, then $p \nmid W_{3 \lambda}$ and since $T_{6 \lambda} \equiv 0(\bmod p)$, we must have $p \mid T_{3 \lambda}$; thus, $p \mid T_{\lambda} S_{\lambda} R_{\lambda}$. If $p \mid T_{\lambda}$, then $p \mid T_{2 \lambda}$ and $\eta=0$; if $p \mid S_{\lambda}$ then $p \mid R_{2 \lambda}$ and $\eta=2$; if $p \mid R_{\lambda}$, then $p \mid S_{2 \lambda}$ and $\eta=1$.

When $p$ is a prime, $p \equiv 1(\bmod 12)$, and $(H \mid p)=1$, we can obtain a further refinement of the results of Theorem 7 . We first require

LemMA 4. If $p \equiv 1(\bmod 12), \alpha=a+b \rho, p \nmid a^{2}-a b+b^{2}, \pi_{p}=$ $r+s \rho$ and $\tau=(a s-b r \mid p)$ (Legendre symbol), then in $Q(\rho)$

$$
\alpha^{(p-1) / 2} \equiv \tau\left(\bmod \pi_{p}\right)
$$

Proof. The proof of this result is completely analogous to the proof given by Dirichlet [1] of a similar result concerning the value of $\alpha^{(p-1) / 2}(\bmod \pi)$, when $\alpha, \pi \in Q(i), i^{2}=1$.

THEOREM 8. Let $p$ be a prime such that $p \equiv 1(\bmod 12)$, $(H \mid p)=1, \pi_{p}=r+s \rho$. If $\tau=(a s-b r \mid p), \nu=\tau(H \mid p)_{4}$, and $\mu=$ $(p-1) / 12$, then one and only one of $W_{\mu}, X_{\mu}, Y_{\mu}, R_{\mu}, S_{\mu}, T_{\mu}$ is divisible by $p$ and that one is given in the table below according to the value of $\nu$ and $\eta$.

\begin{tabular}{|r|c|c|c|}
\hline$\nu$ & 0 & 1 & 2 \\
\hline-1 & $W_{\mu}$ & $Y_{\mu}$ & $X_{\mu}$ \\
\hline 1 & $T_{\mu}$ & $S_{\mu}$ & $R_{\mu}$ \\
\hline
\end{tabular}

Proof. Since $W_{(p-1) / 2}=\alpha^{(p-1) / 2}+\beta^{(p-1) / 2}$ and $\alpha^{(p-1) 2} \beta^{(p-1) / 2} \equiv 1$ $(\bmod p)$, we see that $W_{(p-1) / 2} \equiv 2 \tau\left(\bmod \pi_{p}\right)$ and consequently $W_{(p-1) / 2} \equiv$ $2 \tau(\bmod p)$.

Now

$$
W_{(p-1) / 2}=W_{(p-1) / 4}^{2}-2 H^{(p-1) / 4} ;
$$

thus, $p \mid W_{3 \mu}$ when $\nu=-1$ and $p \mid T_{3 \mu}$ when $\nu=1$.

The remainder of the theorem follows by using reasoning similar to that used in the proof of Theorem 7 .

Using Theorem 7 , we see that if $\eta \neq 0, \sigma=-1$, and if $(p-\varepsilon) / 3$ has no prime divisors which are of the form $6 t-1$, then $\omega_{1}=\omega_{2} / 2$ 
when $\eta=2$ and $\omega_{2}=\omega_{1} / 2$ when $\eta=1$. For suppose $\eta=2, \sigma=-1$ and $2 \lambda=(p-\varepsilon) / 3$. Since $Y_{\lambda} \equiv 0(\bmod p)$ we see that $S_{\lambda} \not \equiv 0(\bmod p)$ and $R_{2 \lambda} \equiv 0(\bmod p)$.

Hence

$$
2 \lambda=\omega_{1}(3 k+1)
$$

or

$$
2 \lambda=\omega_{2}(6 k-1), \quad \text { where } \omega_{1}=2 \omega_{2} .
$$

Since no prime factor of the form $6 t-1$ divides $\lambda$, we must have

$$
2 \lambda=\omega_{1}(3 k+1) \text {. }
$$

If $\omega_{1}=2 \omega_{2}, \lambda=(3 k+1) \omega_{2}$ and $p \mid S_{\lambda}$ which is not so; thus, $\omega_{1}=\omega_{2} / 2$.

5. Primality testing and pseudoprimes. In this section we require the symbol $[A+B \rho \mid C+D \rho]$ of Williams and Holte [7]. In [7] it is shown how this symbol may be easily evaluated. It is also pointed out that if $C+D \rho$ is a prime of $Q(\rho)$, then $[A+B \rho \mid C+D \rho]$ is the cubic character of $A+B \rho$ modulo $C+D \rho$. We are now able to give the main result of this paper.

THEOREM 9. Let $N=2^{n} 3^{m} A-1$, where $n>1, A$ is odd, and $A<2^{n+1} 3^{m}-1$. If $(H \mid N)=-1$ (Jacobi symbol), $[a+b \rho \mid N]=\rho^{\eta}$ $(\eta \neq 0)$, then $N$ is a prime if and only if

$$
X_{L} \equiv 0(\bmod N) \quad \text { when } \quad \eta=1
$$

or

$$
Y_{L} \equiv 0(\bmod N) \quad \text { when } \quad \eta=2 .
$$

Here $L=(N+1) / 6$.

Proof. If $N$ is a prime, $[a+b \rho \mid N]$ is the cubic character of $\alpha H$ modulo $N$; hence, $N \mid X_{L}$ when $\eta=1$ and $N \mid Y_{L}$ when $\eta=2$.

If $N \mid X_{L}$, then $N \mid T_{6 L}$. If $p$ is any prime divisor of $T_{2 L}$ or $T_{3 L}$, then $p$ must divide one of $T_{L}, W_{L}, R_{L}, S_{L}$. From the simple identities which relate $R_{k}, S_{k}, T_{k}$ to $W_{k}, X_{k}, Y_{k}$, we see that if $p \mid X_{L}$, then $p$ must divide two of $R_{L}, S_{L}$, and $T_{L}$, which is impossible; hence $\left(N, T_{2 L}\right)=\left(N, T_{3 L}\right)=1$. Let $p$ be any prime divisor of $N$ and let $\omega=\omega(p)$. We have $\omega \mid 6 L$ but $\omega \nmid 2 L$ and $\omega \nmid 3 L$; thus, $2^{n} \mid \omega$ and $3^{m} \mid \omega$. Since $\omega \mid p \pm 1$, we have

$$
p=2^{n} 3^{m} u \pm 1 \text {. }
$$

Since $N=p S$ for some $S$, we have $S=2^{n} 3^{m} v \pm 1$ and $A=2^{n} 3^{m} u v \pm$ 
$(v-u)$. Now $A$ is odd and $n>1$; hence, one of $u$, $v$ must be even and $A \geqq 2^{n+1} 3^{m}-1$, which is not possible; thus, $N$ is a prime. Similarly, it can be shown that if $N \mid Y_{L}$, then $N$ is a prime.

This criterion for the primality of $N$ can be easily implemented on a computer by making use of the identities

$$
\begin{aligned}
R_{2 k} & =-S_{k}\left(2 R_{k}+S_{k}\right) \\
S_{2 k} & =R_{k}\left(2 S_{k}+R_{k}\right) \\
R_{k+1} & =a R_{k}+b S_{k} \\
S_{k+1} & =(a-b) S_{k}-b R_{k} .
\end{aligned}
$$

The values of $a, b$ can be easily found by trial and then $R_{L}, S_{L}$ determined modulo $N$ by using the above identities in conjunction with a power technique such as that of Lehmer [3].

It is of some interest to determine whether there exist composite values of $N=2^{n} 3^{m} A-1$ such that $A \geqq 2^{n+1} 3^{m}-1,[a+b \rho \mid N]=\rho^{\eta}$, $\eta \neq 0,(H \mid N)=-1$, and

$$
X_{L} \equiv 0(\bmod N) \quad \text { when } \quad \eta=1
$$

or

$$
Y_{L} \equiv 0(\bmod N) \quad \text { when } \quad \eta=2 \quad(L=(N+1) / 6) .
$$

Such values of $N$ can be considered as a type of pseudoprime. In fact, if $N \equiv-1(\bmod 3),[H(a+b \rho) \mid N]=\rho^{\eta}, \sigma=(H \mid N)$, we define $N$ to be an $\alpha$-pseudoprime to base $a+b \rho$ if it divides the appropriate entry of Table 1 with $\lambda=(N+1) / 6$. For example, if $\sigma=-1$, $\rho=2, N$ is an $\alpha$-pseudoprime if

$$
Y_{(N+1) / 6} \equiv 0(\bmod N) \text {. }
$$

A systematic search of all composite $\alpha$-pseudoprimes $\left(<10^{6}\right)$ to base $2+3 \rho$ produced the following:

$$
\begin{array}{lll}
N=5777=53 \cdot 109 & \eta=1, & \sigma=1, \\
N=31877=127 \cdot 251 & \eta=0, & \sigma=-1, \\
N=513197=41 \cdot 12517 & \eta=0, & \sigma=-1, \\
N=915983=47 \cdot 19489 & \eta=1, & \sigma=1 .
\end{array}
$$

None of these has both $\sigma=-1$ and $\eta \neq 0$. Such $\alpha$-pseudoprimes seem to be rather rare; however, they do exist. For example, let $q, p_{1}$, be primes such that $q \equiv 1(\bmod 3), p_{1}=6 q-1$ and select $a, b$ such that $\left[a+b \rho \mid p_{1}\right]=\rho^{2}$ and $\left(H \mid p_{1}\right)=-1$. If $p_{2}$ is prime such that $p_{2} \equiv 13(\bmod 36), \quad\left(p_{2}, p_{1}(2 b-a)\right)=1$ and $Y_{q} \equiv 0\left(\bmod p_{2}\right)$, then $N=p_{1} p_{2}$ is an $\alpha$-pseudoprime to base $a+b \rho$ and 


\section{$N \mid X_{(N+1) / 6}$,}

$(N \mid H)=-1,[a+b \rho \mid N]=\rho$. To prove this we first note that $p_{1} \mid Y_{q}$ and $p_{2} \mid Y_{q}$; hence, $N \mid Y_{q}$. We also have $p_{2} \mid R_{2 q}, p_{2} \nmid S_{q}$ and $p_{2} \nmid R_{2}=$ $Y_{1} S_{1}$; therefore, $\omega_{1}\left(p_{2}\right)=2 q, \quad \omega_{2}\left(p_{2}\right)=4 q$ and $\omega\left(p_{2}\right)=6 q$. Since $\omega\left(p_{2}\right) \mid p_{2}-1$, we see that $12 q \mid p_{2}-1$ and $\left(p_{2}-1\right) / 12 q \equiv 1(\bmod 3)$; consequently, $R_{\left(p_{2}-1\right) / 6} \equiv 0\left(\bmod p_{2}\right),\left(H \mid p_{2}\right)=+1$, and $\left[H(a+b \rho) \mid \pi_{2}\right]=\rho$. Now $p_{1} p_{2}+1 \equiv 0(\bmod 6 q)$ and $\left(p_{1} p_{2}+1\right) / 6 q \equiv-1(\bmod 6)$; hence,

$$
\begin{aligned}
& X_{\left(p_{1} p_{2}+1\right) / 6} \equiv 0\left(\bmod p_{1} p_{2}\right), \\
& \left(H \mid p_{1} p_{2}\right)=\left(H \mid p_{1}\right)\left(H \mid p_{2}\right)=-1 \text {, and } \\
& {\left[\frac{a+b \rho}{p_{1} p_{2}}\right]=\left[\frac{a+b \rho}{p_{1}}\right]\left[\frac{H(a+b \rho)}{\pi_{2}}\right]\left[\frac{H(a+b \rho)}{\bar{\pi}_{2}}\right]=\left[\frac{(a+b \rho)^{2}\left(a+b \rho^{2}\right)}{\bar{\pi}_{2}}\right]} \\
& =\overline{\left[\frac{\left(a+b \rho^{2}\right)^{2}(a+b \rho)}{\pi_{2}}\right]}=\overline{\left[\frac{(a+b \rho)^{2}\left(a+b \rho^{2}\right)}{\pi_{2}}\right]^{-1}}=\rho \text {. }
\end{aligned}
$$

If we put $q=5449, p_{1}=32693, a=2, b=3$, we have $\left(H \mid p_{1}\right)=$ $-1,\left[a+b \rho \mid p_{1}\right]=\rho^{2}$. We also find that the prime 653881 divides $Y_{5449}$; hence, $N=32693 \cdot 653881=21377331533$ is an $\alpha$-pseudoprime to base $2+3 \rho$ and $N \mid X_{(N+1) / 6}$.

6. Acknowledgment. The author gratefully acknowledges the help of the referee in improving the presentation of this material and for correcting many typographical errors.

\section{REFERENCES}

1. G. L. Dirichlet, Demonstration d'une propriété analogue à la loi de réciprocité qui existe entre deux nombres premiers quelconques, J. reine angew. Math., 9 (1832), 379-389.

2. D. H. Lehmer, An extended theory of Lucas' functions, Ann. of Math., (2), 31 (1930), 419-448.

3. - Computer technology applied to the theory of numbers, M.A.A. Studies in Mathematics, 6 (1969), 117-151.

4. P. M. MacMahon, Combinatory Analysis, Chelsea Publishing Co., New York, 1960.

5. H. Riesel, Lucasian criteria for the primality of $N=h \cdot 2^{n}-1$, Math. Comp., 23 (1969), 869-875.

6. H. C. Williams, The primality of $N=2 A 3^{n}-1$, Canad. Math. Bull., 15 (1972), 585-589.

7. H. C. Williams and R. Holte, Computation of the solution of $x^{3}+D y^{3}=1$, Math. Comp., 31 (1977).

Received March 29, 1977 and in revised form November 21, 1977.

The University of Manitoba

Winnipeg, Canada R3T 2N2 



\section{PACIFIC JOURNAL OF MATHEMATICS}

\section{EDITORS}

RICHARD ARENS (Managing Editor)

University of California

Los Angeles, California 90024

C. W. Curtis

University of Oregon

Eugene, OR 97403

C. C. MOORE

University of California

Berkeley, CA 94720

\section{J. DUGUNDJI}

Department of Mathematics University of Southern California Los Angeles, California 90007

R. Finn AND J. Milgram Stanford University Stanford, California 94305

\section{ASSOCIATE EDITORS}

E. F. BeCK ENBACH

B. H. NeUMaNN

F. WOLF

K. Yoshida

\section{SUPPORTING INSTITUTIONS}

UNIVERSITY OF BRITISH COLUMBIA CALIFORNIA INSTITUTE OF TECHNOLOGY UNIVERSITY OF CALIFORNIA MONTANA STATE UNIVERSITY UNIVERSITY OF NEVADA, RENO NEW MEXICO STATE UNIVERSITY OREGON STATE UNIVERSITY UNIVERSITY OF OREGON
UNIVERSITY OF SOUTHERN CALIFORNIA STANFORD UNIVERSITY UNIVERSITY OF HAWAII UNIVERSITY OF TOKYO UNIVERSITY OF UTAH WASHINGTON STATE UNIVERSITY UNIVERSITY OF WASHINGTON 


\section{Pacific Journal of Mathematics \\ Vol. 77, No. $1 \quad$ January, 1978}

Dan Amir, Chebyshev centers and uniform convexity ............... 1

Lawrence Wasson Baggett, Representations of the Mautner group. I ..... 7

George Benke, Trigonometric approximation theory in compact totally

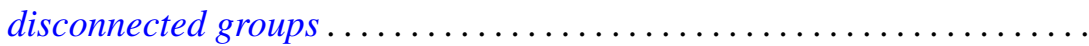

M. Bianchini, O. W. Paques and M. C. Zaine, On the strong compact-ported topology for spaces of holomorphic mappings ..................

Marilyn Breen, Sets with $(d-2)$-dimensional kernels

J. L. Brenner and Allen Kenneth Charnow, Free semigroups of $2 \times 2$

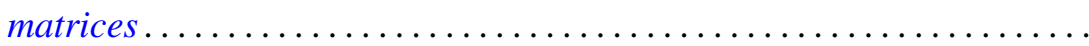

David Bressoud, A new family of partition identities .................

David Fleming Dawson, Summability of matrix transforms of stretchings

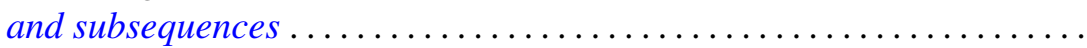

Harold George Diamond and Paul Erdôs, A measure of the nonmonotonicity

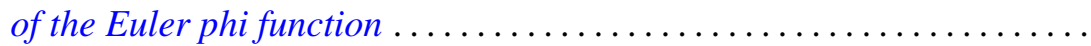

Gary Doyle Faulkner and Ronald Wesley Shonkwiler, Kernel dilation in reproducing kernel Hilbert space and its application to moment

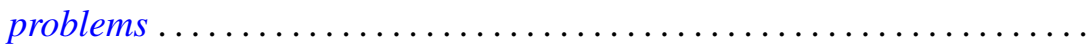

Jan Maksymilian Gronski, Classification of closed sets of attainability in the

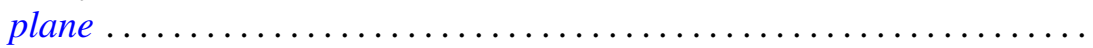

H. B. Hamilton and T. E. Nordahl, Semigroups whose lattice of congruences

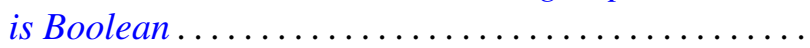

Harvey Bayard Keynes and D. Newton, Minimal $(G, \tau)$-extensions ...

Anthony To-Ming Lau, The Fourier-Stieltjes algebra of a topological

semigroup with involution.

B. C. Oltikar and Luis Ribes, On prosupersolvable groups ...

Brian Lee Peterson, Extensions of pro-affine algebraic groups ...

Thomas M. Phillips, Primitive extensions of Aronszajn spaces ...

Mehdi Radjabalipour, Equivalence of decomposable and 2-decomposable operators. .

M. Satyanarayana, Naturally totally ordered semigroups .

Fred Rex Sinal, A homeomorphism classification of wildly imbedded two-spheres in $S^{3}$

Hugh C. Williams, Some properties of a special set of recurring

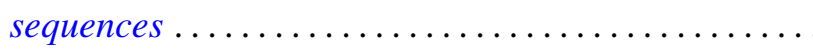

